\title{
Can a High W-6/W-3 Fatty Acid Ratio in The Tissues Predispose Infertility?
}

\author{
Jan Fedacko ${ }^{1, *}$, Viola Vargova ${ }^{1}$, Ram B. Singh ${ }^{2}$, Fabien De Meester ${ }^{2}$, Amit K. Singh ${ }^{3}$, \\ Douglas Wilson ${ }^{4}$ and Suniti M. Dharwadkar ${ }^{5}$
}

\author{
${ }^{I}$ Faculty of Medicine, PJ Safaric University, Kosice, Slovakia; ${ }^{2}$ Tsim Tsoum Institute, Krakow, Poland; ${ }^{3}$ Institute \\ of Medical Sciences, BHU, Varanasi, India; ${ }^{4}$ School of Medicine and Health, Durham University, UK; ${ }^{5}$ Biochemistry \\ Department, S.B. College Science, Aurangabad, India
}

\begin{abstract}
Mediterranean diet appears to be protective and Western diet may be a risk factor for emergence of noncommunicable diseases (NCDS), including infertility. Inflammation is the most important manifestation and risk factor for NCDs which can be prevented by increased intake of Mediterranean foods rich in omega 3 fatty acids (w-3). The biochemical contents of sperm and seminal fluid as well as of egg coating is also imperative in the mechanism of fertilization. Progesterone is known to activate CatSper, (cation channel of sperm) the principal $\mathrm{Ca}^{2+}$ channel of the sperm flagellum which is important in fertilization. The receptors on the external cell membrane to which progesterone binds may be under the influence of various nutrients. The present review is focused on the significance of $w-6$ to $w-3$ ratio on the motility of sperms. Majority of cytoprotective neutraceuticals including w-3 fatty acids and Coenzyme Q 10 (CoQ10) are present in the mitochondria cell membranes. Since composition of the spermatozoa may be important in infertility, this poses the possibility that $\mathrm{w}-6 / \mathrm{w}-3$ ratio of the spermatozoa and seminal fluid can influence progesterone activity and its sensing by the $\mathrm{Ca}^{+2}$ channel.Recent studies demonstrated that the CatSper channel serves as a novel progesterone receptor that can mediate a fast, non-genomic effect of progesterone at the level of the sperm plasma membrane. Further studies are necessary to find out the role of w-6/w-3 ratio and CoQ10 levels on $\mathrm{Ca}^{+2}$ channels of the sperm, in the seminal fluid and ova.
\end{abstract}

Keywords: Fertility, spermatozoa, progesterone, CatSper,w-6/w-3 ratio.

\section{INTRODUCTION}

Recent studies indicate that Mediterranean diet rich in fruits, vegetables, nuts, canola oil, olive oil as well as physical activity, and meditation can decrease inflammation and may be protective against risk of noncommunicable diseases (NCDs). [1-6]. Western diet is characterized with energy dense, refined, ready prepared foods with a high glycemic index (e.g., refined starches bread, biscuits, candies, cornflakes, pizza, potato chips, cola drinks and sugar) and unhealthy lipids (e.g., trans fats, saturated fat, w-6 rich oils) poor in certain phytochemicals and fiber. Such diets have been adopted by an increased number of people and populations in the Western world and in the urban populations of middle income countries in the last few decades [1-4, 7-11]. These diets are known to predispose the epidemic of NCDs; cardiovascular disease, diabetes mellitus, chronic lung diseases, obesity, cancer, autoimmune diseases, rheumatoid arthritis, asthma, infertility and depression [1-6]. These NCDs are associated with increased production of thromboxane A2(TXA2), leucotrienes, prostacyclin, interleukins-1 and -6 , tumor necrosis factor-alpha and C-reactive proteins and oxidative stress [11-15]. Increased dietary intake of w-6 fatty acids, particularly in the presence of low w-3 fatty acids and $\mathrm{CoQ} 10$, is known to enhance all these biomarkers which have adverse proinflammatory effects, resulting in NCDs

*Address correspondence to this author at the Faculty of Medicine, PJ Safaric University, Kosice, Slovakia; Tel: 8805221393;

E-mail janfedacko@hotmail.com including infertility and other reproductive problems [1115]. Recently two independent research groups reported that progesterone potently activates CatSper, the principal $\mathrm{Ca}^{2+}$ channel of the sperm flagellum which is important in fertilization $[16,17]$. Since composition of the sperm membrane appears to be important in infertility, this poses the possibility that the w-6/w-3 ratio and Coenzyme Q10 content of the spermatozoa can influence progesterone activity and its sensing by $\mathrm{Ca}^{+2}$ channels [14, 18-24].

\section{PROGESTERONE AND INFERTILITY}

Infertility affects approximately $10-15 \%$ of reproductiveage couples [5]. In $25 \%$ of cases, infertility is attributable to poor semen quality, the etiology of which is idiopathic [6]. According to a report, sperm concentration had fallen by $40 \%$ over the past 50 years [6]. This finding led to much speculation about the cause and the mechanism of the decline of semen quality. The environment around spermatozoa, including seminal plasma, is thought to play an important role in sperm function $[5,6]$.

Progesterone is a female steroid hormone, produced mainly by the ovaries and the placenta, and supports gestation and embryogenesis through its actions on a wellcharacterized nuclear progesterone receptor. However, the progesterone released by cells surrounding the egg in females can stimulate sperm cells, within the fallopian tubes, and enhance their capability to fertilize the egg $[16,17]$. The effect of environmental factors and dietary changes on progesterone activity has not been given much attention in 
the past. The exact molecular mechanisms underlying how human sperm detects the egg with the help of progesterone has remained elusive.

The probable answer for this mechanism lies in a unique ion channel that senses progesterone, causing an influx of calcium into the sperm cells. A cloud of cumulus cells surrounding the egg release progesterone, prior to fertilization, triggering a calcium influx into the sperm [16]. This flood of calcium causes the sperm to beat their flagella rapidly. This action is necessary for the sperm to penetrate though the egg's protective jelly-like protein coating. Progesterone has also been implicated in providing a chemical gradient by which sperm can navigate toward the egg. However, how calcium is directed to the sperm's calcium channel that senses progesterone is still unknown $[16,17]$. The role of other nutrients in the triggering of calcium and calciummagnesium interactions are also unknown.

The majority of the w-3 fatty acids and CoQ10 are present in the mitochondrial cell membranes. CoQ10 is mainly concentrated in the muscles and organs of the body and is a potent antioxidant and bioenergetic agent. Both the nutrients are cytoprotective. In most human cells, where appropriate, progesterone binds nuclear receptors inside the cell and activates transcription. Sperm, however, are transcriptionally silent, so scientists have searched for a different mechanism by which progesterone activates them, such as a receptor on the external membrane of the cell. These receptors may be under the influence of various nutrients, vitamin A.E.C beta carotene, zinc, copper, selenium, calcium, magnesium, w-3 fatty acids and related enzymes. Composition of the egg's protective jelly-like protein coating and that of sperm membrane, in relation to the $\mathrm{w}-3$ fatty acids, particularly w-6/w-3 ratio appear to be important in the mechanism of fertilization [15-22]. w-3 FA are essential polyunsaturated fatty acids (PUFAs) because it is necessary to acquire them through food consumption. Docosahexaenoic acid (DHA), eicosapentaenoic acid (EPA) and $\alpha$-linolenic acid (ALA) are the main fatty acids which appear to be important for the integrity of plasma lipid membranes. These fatty acids may also influence the functioning of the egg coating in females and sperm membrane and seminal fluid in males. If the protein coating on the egg is less favourable for sperm, it may be difficult for sperm to penetrate and cause fertilization of the egg. Therefore, the biochemical contents of sperm and seminal fluid as well as of egg coating appear to be important in the mechanism of fertilization.

\section{NEW EVIDENCE}

Progesterone acts by regulating gene expression through a well-characterized progesterone nuclear receptor However, the effect of progesterone upon transcriptionally silent spermatozoa remains unexplained and is believed to be mediated by a specialized, non-genomic membrane progesterone receptor. The identity of this non-genomic progesterone receptor and the mechanism by which it causes $\mathrm{Ca}^{2+}$ entry remain fundamental unresolved questions in human reproduction.

Recent studies demonstrate that the CatSper channel or a directly associated membrane protein, serves as a novel progesterone receptor that can mediate a fast, non-genomic effect of progesterone at the level of the sperm plasma membrane $[16,17]$. These results should help to define the physiological role of progesterone and CatSper in sperm, and could lead to the development of new classes of nonhormonal contraceptives. The role of the w-6/w-3 fatty acid ratio of such tissues have been ignored in these studies. Progesterone is released into the oviduct by the cumulus cells which surround the oocyte. In human sperm, progesterone stimulates a $\mathrm{Ca}^{2+}$ increase by a non-genomic mechanism [16]. The $\mathrm{Ca}^{2+}$ signal has been proposed to control chemotaxis, hyperactivation and acrosomal exocytosis of sperm. (Fig. 1). However, the underlying signalling mechanism has remained mysterious and the role of calcium-magnesium exchange as well as the $\mathrm{w}-6 / \mathrm{w}-3$ ratio of fatty acids in the cell membrane of sperm have not been considered or reported. It has been shown that progesterone activates the sperm-specific, $\mathrm{pH}$-sensitive CatSper $\mathrm{Ca}^{2+}$ channel. Both progesterone and alkaline $\mathrm{pH}$ stimulate a rapid $\mathrm{Ca}^{2+}$ influx with almost no latency, incompatible with a signaling pathway involving metabotropic receptors and second messengers. The $\mathrm{Ca}^{2+}$ signals evoked by alkaline $\mathrm{pH}$ and progesterone are inhibited by the Ca channel blockers NNC 55-0396 and mibefradil $[16,17]$. Patch-clamp recordings from sperm reveal an alkaline-activated current carried by mono- and divalent ions that exhibit all the hallmarks of sperm-specific CatSper $\mathrm{Ca}^{2+}$ channels. Progesterone substantially enhances the CatSper current. The alkaline- and progesterone-activated CatSper current is inhibited by both drugs. The results resolve a long-standing controversy over the non-genomic progesterone signaling.

In human sperm, either the CatSper channel itself or an associated protein serves as the non-genomic progesterone receptor. The identification of CatSper channel blockers will greatly facilitate the study of $\mathrm{Ca}^{2+}$ signaling in sperm and help to define further the physiological role of progesterone and CatSper. There is a need to study the biochemistry of the CatSper channel, particularly, w-6/w-3 ratio, calcium, magnesium and CoQ10 levels in related tissues. Progesterone released by cumulus cells surrounding the egg is a potent

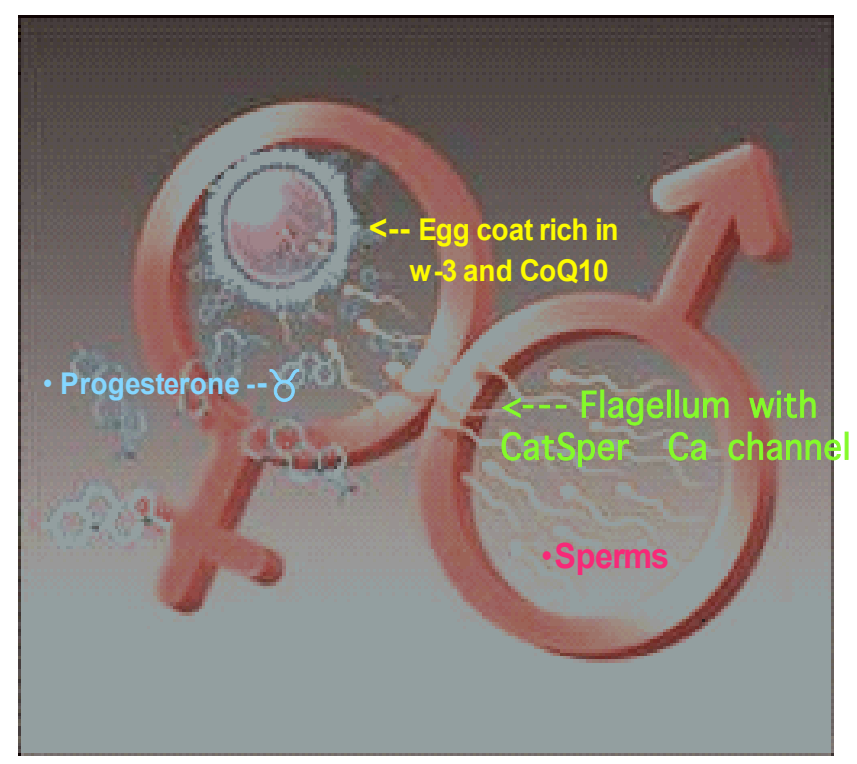

Fig. (1). Ova of female showing liberation of progesterone to attract the sperms during fertilization. 
stimulator of human spermatozoa. It attracts spermatozoa towards the egg and helps them penetrate the egg's protective vestments. Progesterone induces $\mathrm{Ca}^{2+}$ influx into spermatozoa and triggers multiple $\mathrm{Ca}^{2+}$-dependent physiological responses essential for successful fertilization.

A further study elucidated the mechanism of the nongenomic action of progesterone on human spermatozoa by identifying the $\mathrm{Ca}^{2+}$ channel activated by progesterone [17]. By applying the patch-clamp technique to mature human spermatozoa, the authors found that nanomolar concentrations of progesterone dramatically potentiate CatSper, a $\mathrm{pH}$ dependent $\mathrm{Ca}^{2+}$ channel of the sperm flagellum. Human CatSper is synergistically activated by elevation of intracellular $\mathrm{pH}$ and extracellular progesterone. Interestingly, human CatSper can be further potentiated by prostaglandins, but apparently through a binding site other than that for progesterone. Since experimental conditions did not support second messenger signaling, CatSper or a directly associated protein serves as the elusive non-genomic progesterone receptor of sperm. Given that the CatSper-associated progesterone receptor is sperm specific and structurally different from the genomic progesterone receptor, it represents a promising target for the development of a new class of non-hormonal contraceptives which may be related to nutrients. Because of the instantaneous rise of calcium in sperm following exposure to progesterone, it may be concluded that the receptor is not a G-protein coupled receptor or part of a second messenger signaling system. It is possible that progesterone acts directly as a ligand that binds this channel and opens the gates. More research is needed to identify the site of progesterone binding on CatSper and determine what type of ion channel it is, because most of the known $\mathrm{Ca}^{+2}$ channel blockers do not block the $\mathrm{Ca}^{+2}$ channels but they simply delay the opening time of the channels causing decreased entry of $\mathrm{Ca}^{+2}$ into the cell. Once the binding site is identified, it may be possible to design a drug which would not resemble steroids at all and which may be related to a nutrient or herb to achieve inhibition of fertilization (contraception) or facilitating fertilization (conception).

\section{NUTRITIONAL FACTORS AND INFERTILITY}

While in developing countries, men and women are highly fertile, despite coexisting under-nutrition, in developed countries, infertility has become a common social problem which is also increasing now among urban populations of developing countries along with other NCDs [1-7]. The most common male reproductive problems are: motility and quality of the sperm, the decrease in number of the sperm and erectile dysfunction (impotence). There are many factors that can lead to male infertility: stress, excess alcohol intake, smoking, genetic defects, toxic environments, hot baths, spa and sauna and Western diet characterized by a deficiency of w-3 fatty acids, essential amino acids; arginine, coenzyme $\mathrm{Q} 10$, vitamin $\mathrm{E}$ and vitamins $\mathrm{B}$, magnesium, calcium and antioxidants [7-15]. Magnesium is the natural antagonist of calcium and may have a role in opening of the $\mathrm{Ca}^{+2}$ channels via the Ca-Mg-ATPase enzyme. These nutraceuticals appear to be useful in treating male and female infertility. They have the capacity to increase sperm motility and count, and improve the reproductive system and to increase the immune system among both sexes $[16,17]$. Apart from men, deficiency of nutrients can also occur in women leading to poor secretion of progesterone despite normal ovulation. Of these nutraceuticals, w-3 fatty acids and CoQ10, appear to be quite promising [8-15]. The levels of CoQ10 were determined by HPLC in seminal fluid samples obtained from seventy seven patients who performed a standard semen analysis for infertility, previous phlogosis or varicocele (15). CoQ10 was determined in total seminal fluid $(\mathrm{n}=60)$, in seminal plasma $(n=44)$ and in the cell pellet $(n=37)$. The molecule, in total fluid, showed a linear correlation with sperm count and motility. In the pellet of spermatozoa, a trend toward an inverse correlation between CoQ10 (expressed as ng $/ 10^{6}$ cells) and semen parameters could be observed. A different pattern was shown in varicocele patients, in whom, in total fluid, the correlation between CoQ10 and sperm count was preserved, but the one between CoQ10 and sperm motility was lacking.

In a study involving six hundred and ninty infertile men (mean age: 28.5 years) with idiopathic asthenoteratospermia, presenting with male factor infertility for at least one year, daily supplementation with selenium (200 microgram/d) in combination with vitamin E (400 IU) for a period of at least hundred days was found to be associated with significant total improvements in sperm motility, morphology, or both (in $52.6 \%$ of cases), and spontaneous pregnancy $(10.8 \%$ of cases), as compared to no treatment [22]. After fourteen weeks of treatment, only $36.6 \%$ of subjects were found to have no response to treatment. It is possible that supplemental selenium and vitamin E may improve semen quality and have beneficial and protective effects, especially on sperm motility. Their use for the treatment of idiopathic male infertility diagnosed with asthenoteratospermia or asthenospermia in semen analysis will be beneficial.

\section{OMEGA-6 AND OMEGA-3 FATTY ACIDS IN INFER- TILITY}

The lipid composition of the sperm membrane has been shown to exert a significant effect upon the functional quality of spermatozoa. Studies on fatty acid composition of the phospholipids and the ratio of PUFAs to saturated fatty acids (SFAs) of spermatozoa in asthenozoospermic and normozoospermic men were reported [18]. Fatty acid concentration of spermatozoa was determined in fifteen asthenozoospermic and eight normozoospermic semen samples by thin layer chromatography and gas chromatography. The most abundant PUFAs and SFAs in normozoospermic samples were docosahexaenoic acid (DHA $22: 6$ omega 3, $98.5+/-4.5$ nmol per $10^{8}$ spermatozoa, mean $+/-$ SE) and palmitic acid $\left(103+/-17\right.$ nmol per $10^{8}$ spermatozoa) respectively. The mean $+/$ - SE values of DHA and palmitic acid in asthenozoospermic samples were $53.9+/-11.6$ and $145+/-14.7$ nmol per $10^{8}$ spermatozoa) respectively. Compared with normozoospermic samples, asthenozoospermic samples showed lower levels of PUFA and higher amount of SFA. The mean +/- SE ratios of sperm PUFA/SFAs in asthenozoospermic and normozoospermic samples were $0.66+/-0.06$ and $1.45+/-0.16(\mathrm{P}<0.001)$ respectively. This study demonstrates that spermatozoa of asthenozoospermic men have lower levels of DHA- a PUFA compared with palmitic acid - a SFA. (Fig. 2A). This may be contributory to the poor 
motility noted in samples from these men. It is clear that the lipid composition of spermatozoa plays an important role for successful fertilization.

In a further study, fatty acid composition of spermatozoa of normozoospermic, asthenozoospermic, oligozoospermic and oligoasthenozoospermic men were analysed [19]. Spermatozoa from asthenozoospermic $(\mathrm{P}<0.01)$, oligozoospermic $(\mathrm{P}<0.05)$ and oligoasthenozoospermic men $(\mathrm{P}<0.05)$ had lower levels of docosahexaenoic acid (22:6w3, DHA) than those from normozoospermic men. In oligozoospermic and asthenozoospermic men, spermatozoa 18:0 content was higher than that of normozoospermics $(\mathrm{P}<0.01$ and $\mathrm{P}<0.001$, respectively). 18:1w9 was higher in oligoasthenozoospermic and oligozoospermic samples when compared with normozoospermic samples $(\mathrm{P}<0.05$ for both). While from the point of view of total w6 fatty acids, there was no significant difference among the groups, the $\mathrm{w} 6 / \mathrm{w} 3$ ratio was significantly higher in asthenozoospermic samples than in normozoospermic samples $(\mathrm{P}<0.05)$. Monounsaturated fatty acids $($ MFAs) were higher in oligozoospermic samples $(\mathrm{P}<0.05)$ than in normozoospermic samples, PUFAs were lower in asthenozoospermic $(\mathrm{P}<0.01)$, oligoasthenozoospermic $(\mathrm{P}<0.05)$ and oligozoospermic samples $(\mathrm{P}<0.05)$ than in normozoospermic samples. Saturated fatty acids (SFA) were significantly higher in asthenozoospermic $(\mathrm{P}<0.01)$ and oligozoospermic samples $(\mathrm{P}<0.05)$ compared with normozoospermic samples. In correlation analysis, there were significant positive correlations between DHA with sperm motility $(\mathrm{r}=0.53)$, sperm concentration $(\mathrm{r}=0.36)$ and normal sperm morphology $(\mathrm{r}=0.30)$. In addition, there were significant correlations between PUFA with sperm motility $(\mathrm{r}=0.50)$, sperm concentration $(\mathrm{r}=0.35)$, and normal sperm morphology $(\mathrm{r}=0.28)$, and between w6/w3 with sperm motility $(\mathrm{r}=-0.47)$, sperm concentration $(\mathrm{r}=-0.27)$, and normal sperm morphology $(\mathrm{r}=-$ 0.24). These finding suggest that decreased DHA and PUFA, and increased w6/w3 in spermatozoa may be related to infertility in oligo- and/or asthenozoospermic men.

DHA is found in extremely high levels in human ejaculate with the majority occurring in the spermatozoa [20]. However, the relative concentration of DHA and other fatty acids, in blood serum, seminal plasma, and spermatozoa of asthenozoospermic vs. normozoospermic individuals is not known. A recent study reported the phospholipid fatty acid composition of blood serum, seminal plasma, and spermatozoa of normozoospermic men and asthenozoospermic men in order to determine if DHA levels, as well as the levels of other fatty acids, differed. The serum phospholipid DHA levels were similar in the two groups, suggesting similar intakes of dietary DHA. On the other hand, seminal plasma levels of DHA (3.0 vs. $3.7 \%$ ) and total PUFAs (11.8 vs. $13.5 \%$ ) were significantly lower in asthenozoospermic vs. normozoospermic men, respectively, while 18:1 (19.0 vs. $16.8 \%)$ and MUFA (24.2 vs. $21.7 \%$ ) were significantly higher in the asthenozoospermic vs. the normozoospermic men. Spermatozoa from asthenozoospermic men had higher levels of fatty acids 18:1, 20:0, 22:0, 22:1, and 24:0 than sperm from normozoospermic men, and lower levels of 18:0 and DHA (8.2 vs. 13.8\%). Furthermore, total MUFA (19.3 vs. $16.5 \%$ ) was higher and total PUFA (19.0 vs. $24.0 \%$ ), w-3 fatty acids (9.3 vs. $14.6 \%)$, and the ratio of $w-3$ to $w-6$ fatty acids (1.0 vs. 1.6) were lower in the asthenozoospermic men. Therefore, in asthenozoospermic individuals, lower levels of DHA in the seminal plasma, but not in the blood serum, mimic the decreased concentrations of DHA in the spermatozoa. It is likely that the lower concentrations of spermatozoon DHA in these individuals may be due to dietary differences as well as due to some metabolic difference in the asthenozoospermic men.

The effects of dietary supplementation with DHA on DHA levels in serum, seminal plasma, and sperm of asthenozoospermic men as well as on sperm motility were examined in a randomized, double-blind, placebo-controlled manner [21]. Asthenozoospermic men $(\mathrm{n}=28 ;<$ or $=50 \%$ motility) were supplemented with 0,400 , or $800 \mathrm{mg} \mathrm{DHA} / \mathrm{d}$ for 3 months. Sperm motility and the fatty acid composition of serum, seminal plasma, and sperm phospholipid were determined before and after supplementation. In serum, DHA supplementation resulted in decreases in $22: 4 n-6(-30 \%$ in the $800-\mathrm{mg}$ DHA group only) and total w-6 (-6 and $-12 \%$ in the 400- and 800-mg DHA groups, respectively) fatty acids. Increases were noted in DHA (71 and $131 \%$ in the 400 - and 800-mg DHA groups, respectively) and total n-3 fatty acids (42 and $67 \%$ in the $400-$ and $800-\mathrm{mg}$ DHA groups, respectively), and the w-3/n-6 ratio (50 and $93 \%$ in the 400 - and 800-mg DHA groups, respectively). In seminal plasma, DHA supplementation resulted in a decrease in $22: 4 \mathrm{w}-6$ $(-31 \%$ in the $800-\mathrm{mg}$ DHA group only) and an increase in the ratio of w-3 to w-6 (35 and 33\% in the 400- and $800-\mathrm{mg}$ DHA groups, respectively). There were insignificant increases in DHA and total n-3 fatty acids. In sperm, decreases were noted in $22: 4 \mathrm{w}-6(-37$ and $-31 \%$ in the $400-$ and $800-$ mg DHA groups, respectively). There were no other changes. There was no effect of DHA supplementation on sperm motility. The results show that dietary DHA supplementation results in increased serum and possibly seminal plasma--phospholipid DHA levels, without affecting the incorporation of DHA into the spermatozoa phospholipid in asthenozoospermic men. This inability of DHA to be incorporated into sperm phospholipid is most likely responsible for the observed lack of effect of DHA supplementation on sperm motility.

Fatty acid composition of the spermatozoa may be an important determinant of fertility. PUFA composition of the blood plasma and spermatozoa in infertile men with idiopathic oligoasthenoteratozoospermia (OAT) was reported [23]. Eighty-two infertile men with idiopathic OAT and seventy-eight fertile men defined according to semen concentration and proven fertility were enrolled in the study. The semen parameters were assessed according to World Health Organization criteria; two w-6 fatty acids - linoleic acid (LA) and arachidonic acid (AA) and three w-3 fatty acids ALA, EPA and DHA concentrations were measured in blood plasma and spermatozoa; and the seminal plasma enzymatic antioxidant levels of catalase, and superoxide dismutase (SOD), were also assessed. Proven fertile men had higher blood and spermatozoa levels of w-3 fatty aids compared with the infertile patients. The ratio of serum w-6/w-3 fatty acids was significantly higher in infertile $(14.8 \pm 4.3)$ patients compared to fertile controls $(6.3 \pm 2.2)(\mathrm{P}=0.001)$. Additionally, levels of AA were higher and the w-3 index (EPA 
p DHA) was lower in infertile subjects than in fertile controls (all $\mathrm{P}$ values $<0.05$ ). Infertile men had higher mean AA:DHA ratio and AA:EPA $(6.4 \pm 2.9$ and $12.0 \pm 4.9$, respectively) than fertile men $(3.3 \pm 1.8$ and $6.7 \pm 2.6$, respectively) (both $\mathrm{P}=0.001$ ). Ratios of total $\mathrm{w}-6$ to $\mathrm{w}-3 \mathrm{FA}$ under study (AA to DHA and AA to EPA were significantly higher in infertile men (OAT) as compared with the fertile men as depicted in Fig. (2B). A strong negative correlation was found between the AA: DHA and AA:EPA ratios and total sperm count $(\mathrm{r}=0.62, \mathrm{P}=0.001$ and $\mathrm{r}=0.64, \mathrm{P}=0.001$, respectively), sperm motility $(\mathrm{r}=0.63, \mathrm{P}=0.001$ and $\mathrm{r}=0.61, \mathrm{P}=$ 0.001 , respectively), and sperm morphology ( $\mathrm{r}=0.61$, $\mathrm{P}=0.001$, and $\mathrm{r}=0.59, \mathrm{P}=0.002$, respectively). Thus infertile men had lower concentrations of w-3 FAs in spermatozoa than fertile men. These results suggest that further research is necessary to assess the potential benefits of $w-3$ fatty acids supplementation as a therapeutic approach for infertile men. w-3 fatty acids have been implicated in the treatment of all chronic diseases due to their various protective mechanisms [24-28].

In brief, infertility has become a major health problem in association with rapid changes in diet and lifestyle and possibly the use of food packaging materials. It has been observed that during reproduction, nano-molar concentrations of progesterone dramatically potentiate CatSper, a $\mathrm{pH}-$ dependent $\mathrm{Ca}^{2+}$ channel of the sperm flagellum. Human CatSper is synergistically activated by elevation of intracellular $\mathrm{pH}$ and extracellular progesterone. Interestingly, human CatSper can be further potentiated by prostaglandins, but apparently through a binding site other than that of proges-

2A
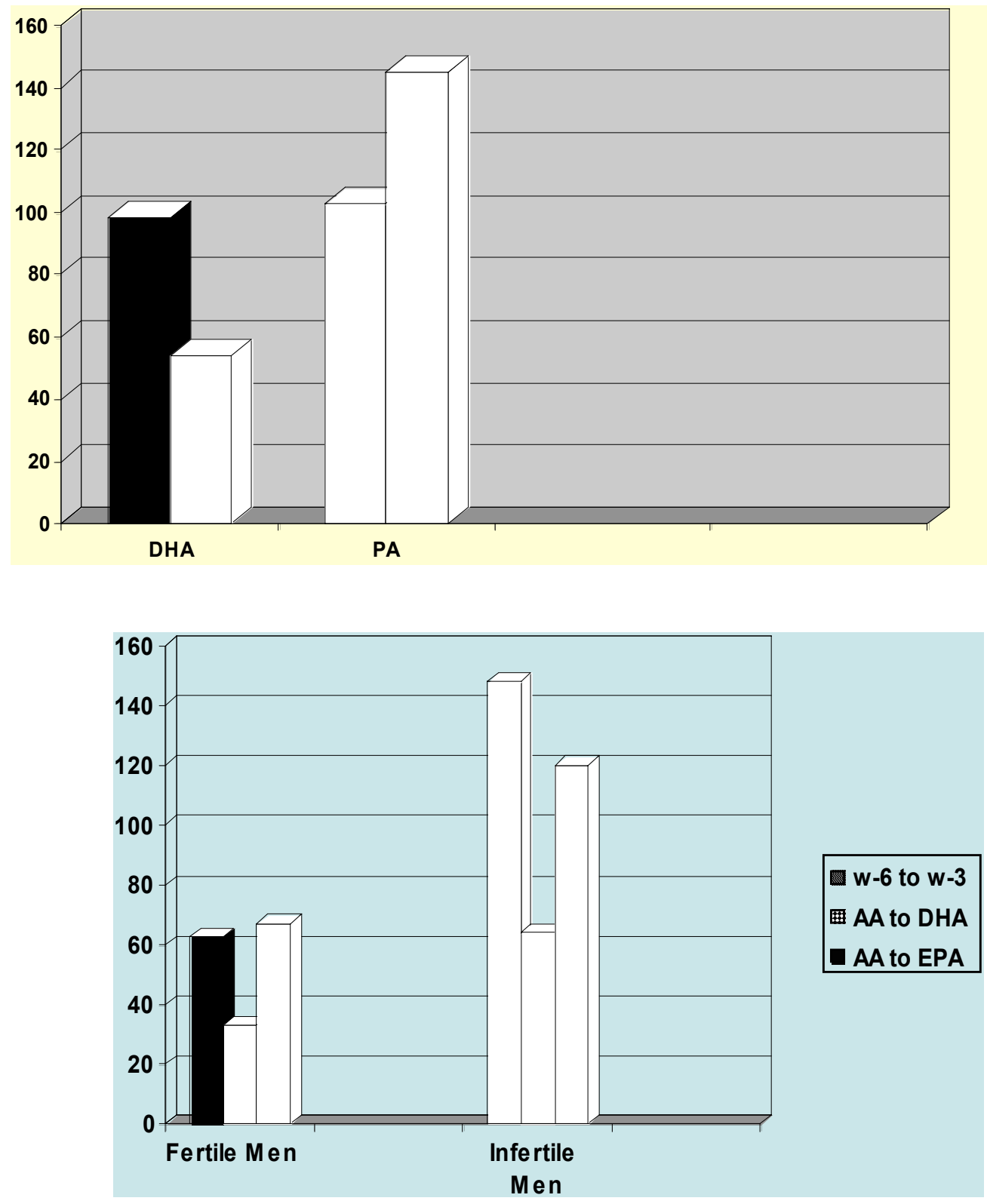

Fig. (2). A. Docosahexaenoic acid (DHA) and Palmitic acid (PA) (nmoles per 100 million spermatozoa) in Normozoospermic (NZSP) and Asthenozoospermic (AZSP) males. (Ref. No. 18). B. Ratios of w-6 to w-3 Fatty acids; Arachidonic acid (AA) to Docosahexaenoic (DHA) and AA to Eicosapentaenoic (EPA) in Fertile and Infertile men (Ref. No 23). 


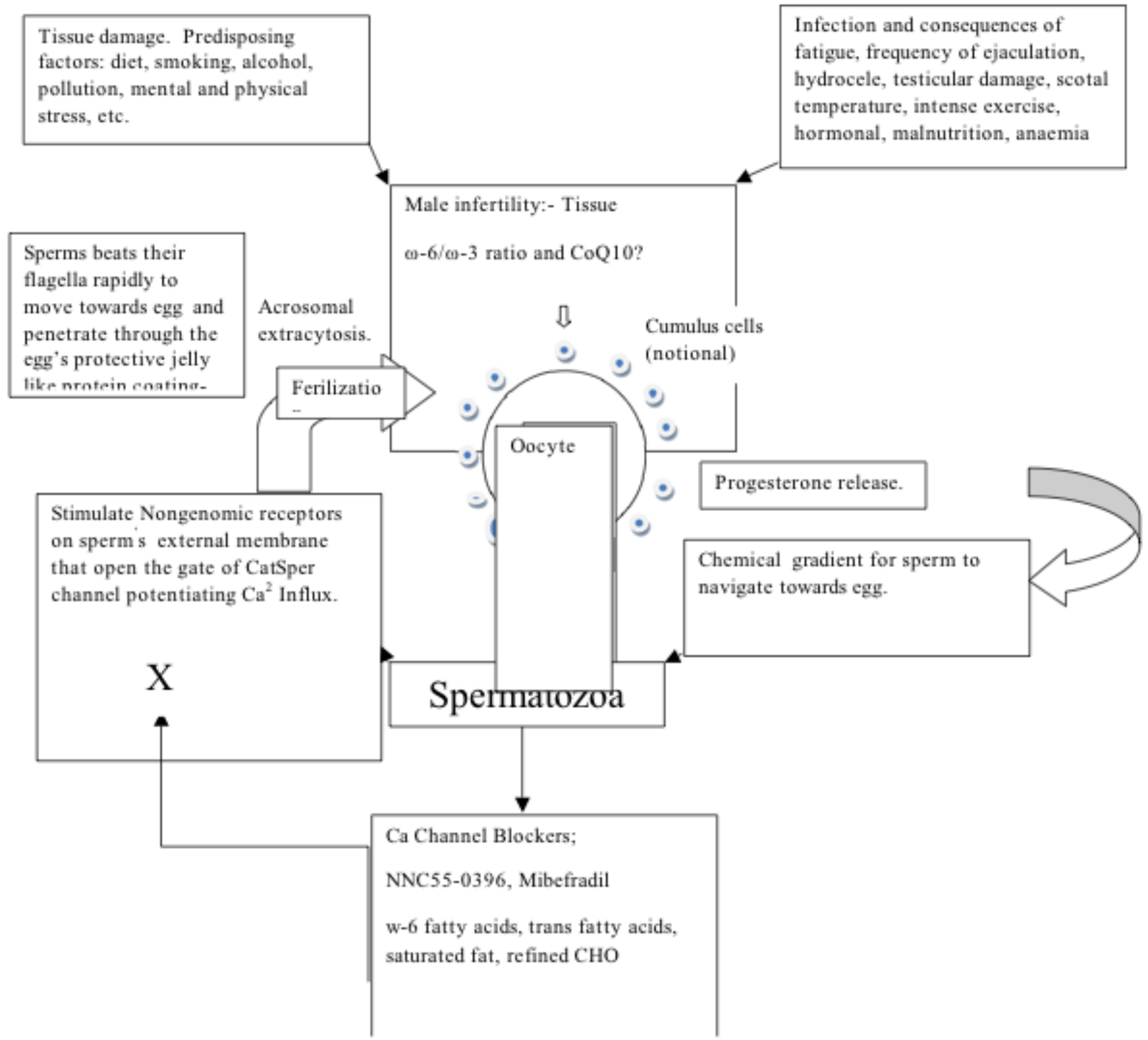

Fig. (3). Effect of environmental factors on ovulation, sperm activity and fertilization via non-genomic progesterone signaling.

terone. Further studies are necessary to find out the role of low and high w-6/w-3 ratio and CoQ10 levels on Ca channels of the sperm, in the seminal fluid and ova, to find out their role on sperm activity of the man and biochemical and clinical characteristics of the egg in relation to progesterone sensing among women (Fig. 3).

\section{ACKNOWLEDGEMENTS}

Acknowledgements are due to International College of Nutrition for providing logistic support to write this article.

\section{REFERENCES}

[1] World Health Organization. Preventing Chronic Disease: A Vital Investment World Heath Organization, Geneva 2005.

[2] Joint WHO/FAO Expert Consultation. Diet, Nutrition and the Prevention of Chronic Diseases, WHO, Geneva, WHO Technical Report Series 2003; 916.
[3] WHO Study Group. Diet, Nutrition and Prevention of Chronic Diseases, WHO, Geneva 1990.

[4] World Health Organization. Diet and Physical Activity: a public Health Priority. World Health Organization, http:/www.who. int/diet physical activity/en/.accessed 2009.

[5] Templeton A. Infertility, epidemiology, etiology and effective management. Health Bull 1995; 53(5): 294-8.

[6] Carlsen E, Giwercman A, Keiding N, Skakkebaek NE. Evidence for decreasing quality of semen during past 50 years. BMJ 1992; 305(6854): 609-13.

[7] Singh RB, Pella D, Mechirova V, et al. Prevalence of obesity, physical inactivity and undernutrition, a triple burden of diseases during transition in a developing economy. The Five City Study Group. Acta Cardiol 2007; 62: 119-27.

[8] Singh RB, Beegom R, Mehta AS, et al. Social class and coronary risk factors and undernutrition: a double burden of diseases among women in five Indian cities. Int J Cardiol 1999; 353: 154-65.

[9] De Meester F. Wild-type land based foods in health promotion and disease prevention: the LDL-CC:HDL-CC model. In Wild Type Foods in Health Promotion and Disease Prevention, eds Fabien De Meester RR, Watson NJ: Humana Press 2008; p. 3-20. 
[10] Vogel RA. Eating vascular biology and atherosclerosis: a lot to chew on. Eur Heart J 2006; 27: 13-14.

[11] Esposito K, Giugiliano D. Diet and inflammation: a link to metabolic and cardiovascular diseases. Eur Heart J 2006; 27: 15-20.

[12] Kelishadi R, Mirghaffari N, Poursafa P, Gidding SS. Lifestyle and environmental factors associated with inflammation, oxidative stress and insulin resistance in children. Atherosclerosis 2009; 203: 311-9.

[13] Simopoulos AP. Is Insulin Resistance Influenced by Dietary Linoleic Acid and Trans Fatty Acids? Free Radic Biol Med1994; 17: 367-72.

[14] Simopolous AP. Genetic variation and dietary response: nutrigenetics/nutrigenomics. Asia Pac J Clin Nutr 2002; 11: S117-28.

[15] Mancini A, Conte B, De Marinis L, et al. Coenzyme Q10 levels in human seminal fluid: diagnostic and clinical implications. MolAspects-Med 1994; 15 (Suppl: s) 249-55.

[16] Strunker T, Goodwin N, Brenker C, et al. The CatSper channel mediates progesterone-induced $\mathrm{Ca} 2+$ influx in human sperm. Nature 2011; 471: 382-6.

[17] Lishko PV, Botchkina IL, Kirichok Y. Progesterone activates the principal $\mathrm{Ca} 2+$ channel of human Sperm. Nature 2011; 471: 387-91.

[18] Aksoy Y, Aksoy H, Altinkayanak K, Aydin HR, Ozkan A. Sperm fatty acid composition in subfertile men. Prosta Leuko Essen Fatty Acids 2006; 75: 75-79.

[19] Tavilani H, Doosti M, Abdi K, Vaisiraigani A, Joshaghani HR. Decreased polyunsaturated and increased saturated fatty acids concentration in spermatozoa from asthenozoospermic males as compared with normozoospermic males. Andrologia 2006; 38: 173-78.
[20] Conquer JA, Martin JB, Tummon I, Watson L, Tekpetey F. Fatty acid analysis of blood, seminal plasma and spermatozoa of normozoospermic and asthenozoospermic males. Lipids 1999; 34: 793-9.

[21] Conquer JA, Martin JB, Tummon I, Watson L, Tekpetey F. Effect of DHA supplementation on DHA Status and sperm motility in asthenozoospermic males. Lipids 2000; 35: 149-54.

[22] Seleniu Moslemi MK, Tavanbakhsh S. Selenium-vitamin E supplementation in infertile men: effects on semen parameters and pregnancy rate. Int J Gen Med 2011; 23(4): 99-104.

[23] Safarinejad MR, Hisseini SY, Dadkhah F, Asgari MA. Relationship of omega-3 and omega- 6 fatty acids with semen characteristics and antioxidant status of seminal plasma: a comparison between fertile and infertile men. Clin Nutr 2010; 29: 100-5.

[24] Simopoulos AP. Importance of the ratio of omega-6/omega-3 essential fatty acids: evolutionary aspects. World Rev Nutr Diet 2003; 92: 1-22

[25] Safarinejad MR. Sperm DNA damage and semen quality impairment after treatment with selective serotonin reuptake inhibitors detected using semen analysis and sperm chromatin structure assay. J Urol 2008; 180(5): 2124-8.

[26] Safarinejad MR. Infertility among couples in a population-based study in Iran: prevalence and associated risk factors. Int J Androl 2008; 31(3): 303-14.

[27] Fraser LR. New insights into possible causes of male infertility. Humanit Rep 1999; 14(Suppl 1): 38-46.

[28] Farooqui AA, Horrocks LA, Farooqui T. Glycerophospholipids in brain: their metabolism, incorporation into membranes, functions, and involvement in neurological disorders. Chem Phys Lipids 2000; 106(1): 1-29. 